\title{
Theoretical study of the energy harvesting of a cantilever with attached prism under aeroelastic galloping
}

\author{
J. Xu-Xu, D. Vicente-Ludlam, A. Barrero-Gil* \\ Aerospace Propulsion and Fluid Mechanics Department, School of Aeronautics, Universidad Politecnica de Madrid, Plaza Cardenal Cisneros 3, E-28040 \\ Madrid. Spain
}

\section{A R T I C L E I N F O}

\section{Article history:}

Available online 24 June 2016

Keywords:

Cantilevered galloping

Energy harvesting

Piezoelectric transducer

\begin{abstract}
A B S T R A T
The aeroelastic galloping of a cantilever with attached prism has recently attracted the attention of several researchers as a way to harvest energy from an airstream. This arrangement is not entirely analogous to that of classical Transverse Galloping (TG) since the instantaneous attitude of the galloping body (prism) with respect to the incident flow depends both on the velocity of the galloping body and wind speed (like in TG) but also on the rotation angle at the cantilever free end. A new governing parameter emerges, namely the ratio of the cross-section length of the prism to the beam length $\delta$, and its effect on the galloping dynamics and power output needs to be studied. To this end, a theoretical model is here developed where the influence of $\delta$ is considered.
\end{abstract}

\section{Introduction}

Flow-induced oscillations by Transverse Galloping (TG) were pointed out by Barrero-Gil et al. [1] as a potential source for energy harvesting from an airstream. TG is a fluid-elastic instability that appears in some elastic bluff bodies when the velocity of the incident flow exceeds a critical value. Then, oscillatory motion (transverse to the flow) develops with increasing amplitude until the energy dissipated per cycle by mechanical damping balances the energy input per cycle from the flow (for a detailed introduction to TG the reader is referred to Parkinson [2], or Paidoussis et al. [3]). If the geometry of the body and the elastic properties are appropriate, the TG instability may appear at low flow velocities and with large excitation amplitudes, making TG a very promising way to harvest energy successfully $[1,4,5]$.

Barrero-Gil et al. [1] made an analytical treatment to give the level of mechanical power extraction as a function of the geometry of the cross-section of the galloping body, its mechanical parameters, and flow velocity. Findings like the maximum efficiency achievable or the wind speed at which this maximum occurs were reported. Since then, several researches have studied how to implement the concept in a real energy harvester, with emphasis in low power generation systems, of the order of milli-Watts or tens of milli-Watts (see, for example, Sirohi and
Mahadik [6,7], Zhao et al. [8], Yang et al. [9], Xu-Xu et al. [10]), with characteristic dimensions of the order of centimeters. They have been focused on experimental arrangements where a rigid galloping body is fixed to the free end of a cantilevered beam (see Fig. 1). For electricity conversion piezoelectric sheets are usually attached to the base of the beam. Under the effect of an airstream, for high enough air speed, oscillations by galloping take place and the induced strain in the piezoelectric patches produces an electrical current which is dissipated at the electrical load $R_{I}$ (see, for example, Yang et al. [9]). However, in this cantilevered arrangement the situation is not entirely analogous to that of pure TG analyzed in Barrero-Gil et al. [1], since the instantaneous attitude of the galloping body with respect to the incident flow depends on the velocity of galloping body and wind speed (like in TG) but also on the rotation angle at the beam free end (see Fig. 1; Kluger et al. [11]). A new governing parameter appears, namely the ratio of the cross-section characteristic length $D$ to the cantilever beam length $L_{b}$, defined as $\delta=3 D /\left(2 L_{b}\right)$, and its role on the dynamics of the body and electrical power output should be studied. With this idea in mind, we present here a theoretical model of a generic energy harvester where the galloping body is cantilevered mounted. Quasi-steady conditions are assumed to model aerodynamic forces and a kinematic relationship is introduced for the instantaneous angle of attack where rotation of the beam is considered. An equivalent circuit model is employed for the piezoelectric sheets. The mathematical model is approximately solved by applying the standard Harmonic Balance Method, and analyzed in detail. The analysis is focused on the 

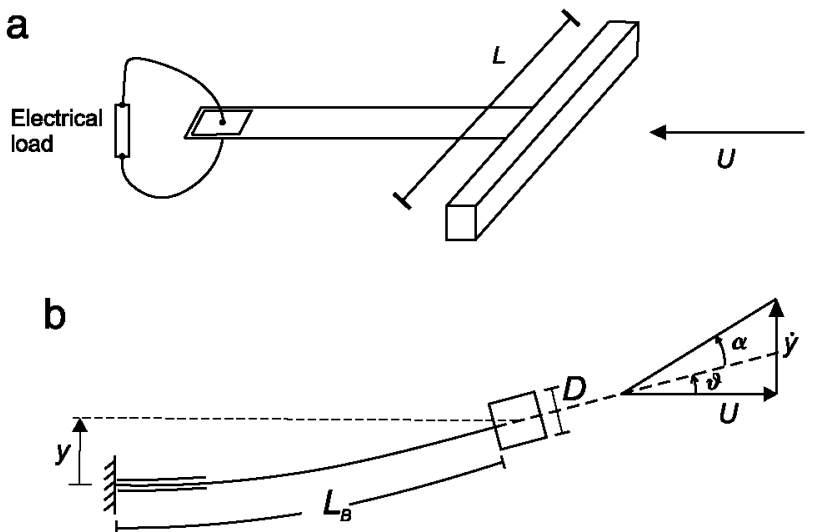

Fig. 1. (a) Typical arrangement piezoelectric energy harvesting from galloping of a cantilevered prism. (b) One-degree-of-freedom model: vertical displacement of the rigid bluff body.

impact of the system dimensionless parameters on the efficiency of energy harvested. As a novelty, the analysis allows to see clearly that $\delta$ has a negative impact into the electrical power output.

First of all, in Section 2, an electro-aero-elastic model is introduced. An analytical approximate solution is found in Section 3 that allows us to get physical insight and to discuss the influence of the length of the beam and other governing parameters on both the galloping body dynamics and electrical power. Analytical predictions are compared with experimental results from Zhao et al. [8] in Section 4. Good agreement is found. Finally, concluding remarks are drawn in Section 5.

\section{Theoretical model}

Let us introduce a one-degree-of-freedom model to describe the transverse displacement of the prism shown in Fig. 1. It is based on the equilibrium between inertia, damping, and stiffness forces, as well as vertical aerodynamic force, and the electromechanical force induced by the piezoelectric transducer. That is,

$m \ddot{y}+c \dot{y}+k y=\frac{1}{2} \rho U^{2} D L C_{Y}-F_{p}$,

where $y$ denotes the transverse position of the prism, $m$ is the equivalent mass of the prism, $c$ is an equivalent damping constant, $k$ is the equivalent stiffness constant, $\rho$ is the fluid density, $U$ is the undisturbed velocity of the incident flow, $D$ the side length of the prism's cross-section and $L$ its length, $C_{Y}$ is the instantaneous aerodynamic force coefficient in the transverse direction to the incident flow, and $F_{p}$ is the electromechanical force in the $y$ direction due to the piezoelectric effect. Finally, the dot symbol stands for differentiation with respect to physical time $t$.

The equivalent (or effective) mass of the prism is given by the prism mass plus the effective mass of cantilever beam. The effective mass of cantilever beam can be approximated as 0.25 times the mass of the cantilever beam [12]. The equivalent damping and stiffness constants can be obtained experimentally from a free decay tests in absence of fluid flow by measuring the decay rate of the amplitude and frequency of oscillations.

Note that, for the sake of simplicity, damping and stiffness forces have been considered linear, which is a realistic approximation when transverse displacements of the prism are small compared to the length of the beam.

\subsection{Aerodynamic force}

In order to describe $C_{Y}$, the quasi-steady hypothesis is usually resorted to (see Paidoussis et al. [2]), since galloping is typically a low-frequency oscillation phenomenon where the characteristic timescale of the prism oscillation (of order $2 \pi(\mathrm{m} / \mathrm{k})^{1 / 2}$ ) is much larger than the characteristic timescale of the flow (of order $D / U)$. Then, the aerodynamic force is only dependent on the instantaneous attitude of the prism with respect to the incident flow, which can be described by the effective angle of attack $\alpha$. From Fig. 1(b),

$\tan (\alpha+\theta)=\frac{\tan \alpha+\tan \theta}{1-\tan \alpha \tan \theta}$,

where $\theta$ is the rotation angle at the free end of the beam; $\alpha$ and $\theta$ are positive in the counterclockwise direction. Assuming that $\alpha$ and $\theta$ are small it follows that

$\tan (\alpha+\theta) \simeq \tan \alpha+\tan \theta$.

For a uniform cantilevered beam, $\theta=3 y /\left(2 L_{b}\right)$ (see Kluger et al. [11]), where $L_{b}$ is the length of the beam. In addition, $\tan (\alpha+\theta)=$ $\dot{y} / U$ so it follows that

$\tan \alpha \simeq \frac{\dot{y}}{U}-\frac{3 y}{2 L_{b}}$.

For our theoretical analysis, to maintain a compromise between development complexity and accuracy, a cubic polynomial can be considered enough (see Blevins [13, p. 130]) to approximate the vertical aerodynamic force coefficient dependence with $\tan \alpha$, so that

$C_{Y}=a_{1} \tan \alpha+a_{3}(\tan \alpha)^{3}$,

where $a_{1}(>0)$ and $a_{3}(<0)$ are the empirical coefficients to fit by a polynomial the $C_{Y}$ versus $\tan (\alpha)$ dependence measured in static tests (normally in wind tunnel). The values of a 1 and a3 depend on the cross-section geometry of the prism. We refer the reader to Blevins [13], Bokaian and Geoola [14] or Barrero-Gil et al. [1] in order to obtain more information and typical values. Then, the aerodynamic force coefficient is

$C_{Y}=a_{1}\left(\frac{\dot{y}}{U}-\frac{3 y}{2 L_{b}}\right)+a_{3}\left(\frac{\dot{y}}{U}-\frac{3 y}{2 L_{b}}\right)^{3}$,

which can be simplified to

$C_{Y}=a_{1}\left(\frac{\dot{y}}{U}-\frac{3 y}{2 L_{b}}\right)+a_{3}\left(\left(\frac{\dot{y}}{U}\right)^{3}+\frac{27 y^{2} \dot{y}}{4 L_{b}^{2} U}\right)$,

if nonlinear stiffness terms are neglected, which makes sense since their effect in the overall response is expected to be small when the bluff body is under the action of light fluids (airstreams). Let us discuss this point in detail by comparing the nonlinear stiffness fluid force $\widetilde{F}_{Y}$

$\widetilde{F}_{Y}=\frac{1}{2} \rho U^{2} D L\left(\frac{27 y^{3}}{8 L_{b}^{3}}-\frac{9 \dot{y}^{2} y}{2 U^{2} L_{b}}\right)$,

and the stiffness force $F_{s}=k y$. That is

$\frac{\widetilde{F}_{Y}}{F_{S}}=\frac{\rho U^{2} D L}{2 k}\left(\frac{27 y^{2}}{8 L_{b}^{3}}-\frac{9 \dot{y}^{2}}{2 U^{2} L_{b}}\right)$.

Taking $y \sim A$, where $A$ is the steady-state amplitude of oscillations, $\dot{y} \sim A \omega_{N}$ where $\omega_{N}^{2}=k / m$ is the natural frequency of oscillations, it follows that

$\frac{\widetilde{F}_{Y}}{F_{s}} \sim \frac{a_{3}}{2 m^{*}}\left(A^{* 2} \delta^{3} U^{* 2}-3 A^{* 2} \delta\right)$,

where $m^{*}=m /\left(\rho D^{2} L\right)$ is the mass ratio, $A^{*}=A / D$ is the normalized steady state amplitude of oscillations, $\delta=3 D /\left(2 L_{b}\right)$, and $U^{*}=$ $U /\left(\omega_{N} D\right)$ is the reduced velocity. Note that nonlinear stiffness fluid force terms are expected to be negligible for large $m^{*}$, which is a common situation when the fluid is light (airstreams for example). 


\subsection{Piezoelectric force}

An equivalent circuit is usually employed to build a linear lumped-model of the piezoelectric sheet (Ertuk et al. [15]). Then

$C_{p} \dot{v}+\frac{v}{R_{L}}+\theta \dot{y}=0$

and

$F_{p}=-\theta v$,

where $v$ is the voltage across the electrical resistance, $C_{p}$ is the equivalent capacitance of the piezoelectric sheets, $\theta$ is an electromechanical coupling constant, and $R_{L}$ the electrical load resistance. Let us consider a steady-state of sinusoidal oscillations, that is

$y=A \sin \omega t$,

and,

$v=v_{0} \sin (\omega t+\phi)$,

where $A$ and $\omega$ are, respectively, the amplitude and frequency of oscillations, $v_{0}$ the voltage amplitude and $\phi$ the phase delay between voltage and transverse displacement of the beam. Substituting Eqs. (13) and (14) into Eq. (11) and equating sine and cosine terms it follows that

$v_{0} \sin \phi=-\frac{\theta R_{L} A \omega}{1+C_{p}^{2} R_{L}^{2} \omega^{2}}$,

and

$v_{0} \cos \phi=-C_{p} R_{L} \omega \frac{\theta R_{L} A \omega}{1+C_{p}^{2} R_{L}^{2} \omega^{2}}$.

Since $\dot{y}=A \omega \cos (\omega t)$ it follows from Eq. (12) and Eqs. (15) and (16) that the piezoelectric force can be split into a damping term and a stiffness one

$F_{p}=c_{E} \dot{y}+c_{E} C_{p} R_{L} \omega^{2} y$,

with

$c_{E}=\frac{\theta^{2} R_{L}}{\left(1+C_{p}^{2} R_{L}^{2} \omega^{2}\right)}$.

\subsection{Dimensionless equation}

Taking into account Eqs. (7) and (17), Eq. (1) can now be rewritten in dimensionless form as

$$
\begin{aligned}
& Y^{\prime \prime}+2 \zeta Y^{\prime}+Y=\frac{U^{* 2} a_{1}}{2 m^{*}}\left(\frac{Y^{\prime}}{U^{*}}-\delta Y\right) \\
& +\frac{U^{* 2} a_{3}}{2 m^{*}}\left(\frac{Y^{\prime 3}}{U^{* 3}}+\frac{3 \delta^{2} Y^{2} Y^{\prime}}{U^{*}}\right)-2 \zeta_{E} Y^{\prime}-2 \zeta_{E} \beta \omega^{* 2} Y,
\end{aligned}
$$

where comma stands differentiation with respect to the dimensionless time, $\tau=\omega_{N} t, Y=y / D$ is the normalized displacement, $\zeta=c /\left(2 m \omega_{N}\right)$ is the dimensionless parasite mechanical damping, $m^{*}=m /\left(\rho D^{2} L\right)$ is the mass ratio, $\delta=3 D /\left(2 L_{b}\right), U^{*}=U / \omega_{N} D$ the reduced velocity, and $\zeta_{E}$ is a dimensionless piezoelectric damping coefficient, given by

$\zeta_{E}=\frac{\theta^{2} R_{L}}{2 m \omega_{N}\left(1+C_{p}^{2} R_{L}^{2} \omega^{2}\right)}$,

$\beta=R_{L} C_{p} \omega_{N}$, and $\omega^{*}=\omega / \omega_{N}$.

The last term of Eq. (19) $\left(2 \zeta_{E} \omega^{* 2} Y\right)$ contributes to the stiffness component of the dynamic equation. When comparing the order of magnitude of this term with the stiffness leading term (of order unity), it is noted that it can be neglected in practical situations since $2 \zeta_{E} \beta \omega^{* 2} \ll 1$. Let us see, $2 \zeta_{E} \beta \omega^{* 2}$ can be rewritten, taking into account the definition in Eq. (20), as:

$2 \zeta_{E} \beta \omega^{* 2}=\frac{\theta^{2}}{m \omega_{N}^{2} C_{p}} \frac{\beta^{2} \omega^{* 2}}{1+\beta^{2} \omega^{* 2}}$.

The second term, $\beta^{2} \omega^{* 2} /\left(1+\beta^{2} \omega^{* 2}\right)$, is always under unity. Let us check the first term $\theta^{2} /\left(m \omega_{N}^{2} C_{p}\right)$ :

- The value of the electromechanical coupling constant $\theta$ is dictated by the state of art of the piezoelectric sheets, and it is or the order $10^{-4} \mathrm{~N} / \mathrm{V}$ nowadays.

- $C p$ is also dictated by the state of art of the piezoelectric sheets, it is about $10^{-7} \mathrm{~F}$ for commercial piezoelectric sheets.

- $m \omega_{N}^{2}$ is equal to the cantilever beam stiffness constant, which depends on the cantilever beam elastic properties and geometric characteristics (cross-section and length of the beam). It takes often a value of the order of $100 \mathrm{~N} / \mathrm{m}$ (see for example, experiments of Zhao et al. [8]).

These parameters above yield that $2 \zeta_{E} \beta \omega^{* 2} \sim 10^{-3}$. Based on that, we conclude, unless the stiffness of the cantilever beam is very low, that the last term of Eq. (19) can be neglected.

As can be seen in Eq. (19), at this level of approach, for $\delta \neq 0$ the aerodynamic force introduces into the dynamics of the prism an additional linear stiffness term as well as a nonlinear damping term. When $\delta \rightarrow 0$, Eq. (19) tends to that of pure TG (see BarreroGil et al. [1]).

\subsection{Electrical power}

The mean electrical power $P_{E}$ dissipated at the electrical load is given by,

$$
\begin{aligned}
P_{E} & =\frac{1}{T} \int_{0}^{T} \frac{v^{2}}{R_{L}} d t \simeq \frac{1}{T} \int_{0}^{T} \frac{\left(v_{0} \sin \phi\right)^{2}}{R_{L}} d t \\
& =\frac{1}{T} \int_{0}^{T} 2 m \omega_{N} \zeta_{E} \dot{y}^{2} d t,
\end{aligned}
$$

where it has been assumed that $v_{0} \cos \phi \ll v_{0} \sin \phi$ (note that $v_{0} \cos \phi=C_{p} R_{L} \omega v_{0} \sin \phi$ and for practical situations $C_{p} R_{L} \ll 1$ ); $T$ is a period time for averaging. An alternative way to arrive to the same result is to note that the power dissipated at the piezoelectric sheet is given by

$P=\frac{1}{T} \int_{0}^{T} F_{p} \dot{y} d t=\frac{1}{T} \int_{0}^{T} c_{E} \dot{y}^{2} d t+\frac{1}{T} \int_{0}^{T} c_{E} C_{P} R_{L} \omega^{2} y \dot{y} d t$

When non-linearity in Eq. (19) is small (high $m^{*}$ which is usual in aero-elastic situations) a steady-state of sinusoidal oscillation is expected so that the last term of Eq. (23) disappears and

$P=\frac{1}{T} \int_{0}^{T} c_{E} \dot{y}^{2} d t=\frac{1}{T} \int_{0}^{T} 2 m \omega_{N} \zeta_{E} \dot{y}^{2} d t$,

which coincides with the expression given in Eq. (22).

An efficiency factor $\eta$ can be introduced relating the mean electrical power dissipated with the total power in the flow per frontal area of the prism

$\eta=\frac{P_{E}}{\frac{1}{2} \rho U^{3} D L}$.

\section{Analytical solution}

\subsection{Dynamic response}

Eq. (19) contains 7 dimensionless parameters $\left(m^{*}, \zeta, \zeta_{E}, U^{*}\right.$, $\left.\delta, a_{1}, a_{3}\right)$, meaning that the solution manifold exists inside a 
7-dimensional space. To gather a better understanding in this 7-dimensional space, the use of a theoretical analysis able to yield an analytical prediction, valid within the assumptions made, is deemed to be beneficial because it clearly identifies the role in the dynamics of any of the governing parameters. Eq. (19) allows an analytical approach when the nonlinear terms are small compared to the linear ones. That is when $m^{*} \gg 1$, which is a usual condition when the fluid medium is air. In this case, solution to the nonlinear Eq. (19) is close to that of its linear version and thus one may assume that the steady movement response is sinusoidal, $Y=A^{*} \sin \left(\omega^{*} \tau\right)$, where $\omega^{*}=\omega / \omega_{N}$ and $A^{*}=A / D$ being $A$ the amplitude of steady oscillations. In addition, if one takes into account that $\cos ^{3}\left(\omega^{*} \tau\right) \approx 3 \cos \left(\omega^{*} \tau\right) / 4$, (i.e. higher order terms in $\cos \left(3 \omega^{*} \tau\right)$ are neglected), equating sine and cosine terms, after some algebra one gets

$\omega^{* 2}=1+\frac{a_{1} U^{\star 2} \delta}{2 m^{*}}=k_{1}$,

$A^{* 2}=\frac{4}{3} \frac{1}{\frac{a_{3} \omega^{* 2}}{U^{*}}+U^{*} \delta^{2} a_{3}}\left(4 m^{*}\left(\zeta+\zeta_{E}\right)-a_{1} U^{*}\right)$

where $k_{1}$ is a parameter introduced for convenience. Eq. (26b) can be written in an explicit way if Eq. (26a) is taken into account,

$A^{* 2}=\frac{4 U^{*}}{3 a_{3}}\left(4 m^{*}\left(\zeta+\zeta_{E}\right)-a_{1} U^{*}\right) k_{2}$,

where $k_{2}=\left(1+\frac{a_{1} U^{* 2} \delta}{2 m^{*}}+U^{* 2} \delta^{2}\right)^{-1}$ has been introduced for convenience. The first point to note is that the dynamics of the cantilevered system tends to that of pure TG (see Barrero-Gil et al. [1]) for long beams ( $\delta \ll 1)$, since the correction factors $k_{1}$ and $k_{2}$ tend to 1 . Second, the reduced velocity at which galloping starts $U_{\mathrm{g}}^{*}$ is given by the point at which $A^{*}=0$, that is

$U_{g}^{*}=\frac{4 m^{*}}{a_{1}}\left(\zeta+\zeta_{E}\right)=\frac{4 m^{*}}{a_{1}}\left(\zeta+\frac{\theta^{2} R_{L}}{2 m \omega_{N}\left(1+C_{p}^{2} R_{L}^{2} \omega_{N}^{2} k_{1}\right)}\right)$,

which indicates that galloping occurs at lower velocities as $\delta$ increases since $k_{1}$ increases too. Third, $\delta$ increases the frequency of oscillations $\left(k_{1}>1\right)$ and diminishes the amplitude of oscillations $\left(k_{2}<1\right)$, more strongly as $m^{*}$ is lower. Finally, for large reduced velocities $A^{*}$ tends to an asymptotic finite value, whereas in pure TG the amplitude of oscillations grows with the reduced velocity without limit. The asymptotic value is given by

$A_{\infty}^{*}=\left(-\frac{8 m^{*} a_{1}}{3 a_{3} \delta\left(a_{1}+2 m^{*} \delta\right)}\right)^{1 / 2}$,

which, as expected, tends to infinite when $\delta$ tends to zero (large beams). Note also that $A_{\infty}^{*}$ does not depend on the electrical properties (piezoelectric sheets properties and electrical resistance).

For very large $m^{*}$ (say, greater than 100 ) and $a_{1} U^{* 2} \delta / m^{*} \ll 1$, which are usual conditions when the fluid medium is air, $k_{2} \approx(1+$ $\left.U^{* 2} \delta^{2}\right)^{-1}$ which indicates that unless $U^{*} \delta$ is small, the amplitude of oscillations is significantly reduced with respect to the case of pure TG. For instance, for $U^{*} \delta$ of order unity the amplitude of galloping oscillations in cantilever arrangement is expected to be of the order of half times the amplitude predicted by pure TG model $\left(k_{2} \sim 1 / 2\right)$. This indicates that pure TG modeling is not adequate for those situations in which $U^{*} \delta \sim 1$.

Finally, the asymptotic limit for amplitude of oscillations when $m^{*}$ is very large is $A_{\infty}^{*} \approx\left(-4 a_{1} /\left(3 a_{3} \delta^{2}\right)\right)^{1 / 2}$. A word of caution is needed here. A main assumption was made during the analysis with respect to the angle of attack, since it was assumed that $\dot{y} / U \sim A^{*} / U^{*}<\alpha_{L I M}$ and $3 y /(2 L) \sim \delta A^{*}<\alpha_{L M}$, meaning $\alpha_{I M M}$ an angle at which the approximation $\tan \left(\alpha_{I M}\right) \simeq \alpha_{I M}$ is still reasonable. In order to fulfill the assumption made, these conditions should be checked. Obviously for situations where this assumption is not valid, either a higher order of approximation or a numerical solution of the system given by Eqs. (1), (2), (5), (8), and (9) is needed. In addition, it should be noted that the elastic model of the cantilever beam is give only by an equivalent bending stiffness and neither torsional nor buckling effects are considered. When $\delta$ is very small, elastic response of the cantilever is expected to be more complex with parasitic torsion motions and a more refined elastic model could be needed.

\subsection{Electrical power}

At the steady-state of harmonic oscillations, from Eq. (22) one may get

$P_{E}=m \omega_{N}^{3} D^{2} \zeta_{E} A^{* 2} \omega^{* 2}$,

and therefore from Eqs. (26a) and (27)

$P_{E}=m \omega_{N}^{3} D^{2} \zeta_{E} \frac{4 U^{*}}{3 a_{3}}\left(4 m^{*}\left(\zeta+\zeta_{E}\right)-a_{1} U^{*}\right) k_{1} k_{2}$

This expression gives the electrical power as a function of the geometrical $\left(a_{1}, a_{3}\right)$ and mechanical $\left(m^{*}, \zeta\right)$ properties of the prism, piezoelectric sheet properties $\left(\zeta_{E}\right)$, flow speed $\left(U^{*}\right)$, and length aspect of the beam $(\delta)$. Not that it may be useful for designing purposes in order to optimize the electrical power output by choosing appropriately the mechanical properties and the electrical resistance.

The efficiency can be now obtained from Eqs. (25) and (31),

$\eta=\frac{8 m^{*} \zeta_{E}}{3 a_{3} U^{* 2}}\left(4 m^{*}\left(\zeta+\zeta_{E}\right)-a 1 U^{*}\right) k_{1} k_{2}$

It is interesting to note that

$k_{1} k_{2}=\frac{1+\frac{a_{1} U^{* 2} \delta}{2 m^{*}}}{1+\frac{a_{1} U^{* 2} \delta}{2 m^{*}}+U^{* 2} \delta^{2}}=\left(1+\frac{U^{* 2} \delta^{2}}{1+\frac{a_{1} U^{* 2} \delta}{2 m^{*}}}\right)^{-1}$

recovers the effect due to the cantilever, and takes a value lower than 1 for $\delta>0$, meaning that there is a drop in the electrical power associated to the cantilever arrangement. For aero-elastic situations in which $m^{*} \gg 1, k_{1} k_{2}$ tends to $\left(1+\delta^{2} U^{* 2}\right)^{-1}$.

From the practical side, it may be of interest to know the maximum achievable efficiency $\eta_{\max }$ for a given configuration as well as the reduced velocity (the wind speed) at which the efficiency is maximum $U_{\eta \text { max }}^{*}$. This can be done by setting $\partial \eta / \partial U^{*}=0$ from Eq. (32), and solving. If $m^{*} \gg 1$ (aeroelastic situation) one arrives to the following equation

$U^{*}-2 U_{g}^{*}-\delta^{2} U^{* 2}\left(4 U_{g}^{*}-3 U^{*}\right)=0$,

which should be solved to find $U_{\eta \max }^{*} ; U_{g}^{*}=4 m^{*}\left(\zeta+\zeta_{E}\right) / a_{1}$ is the reduced velocity at which galloping starts. We have not found an amenable explicit expression for $U_{\eta \max }^{*}$ but a simple analysis of Eq. (34) shows that in the limit $\delta \longrightarrow 0$ then $U_{\eta \max }^{*}$ tends to $2 U_{g}^{*}$. On the contrary, when $\delta \longrightarrow \infty$ then $U_{\eta \max }^{*}$ tends to $4 U_{g}^{*} / 3$. For other conditions of the cantilever beam's length $4 U_{g}^{*} / 3<U_{\eta \max }^{*}<2 U_{g}^{*}$. It is proven analytically that $U_{\eta \max }^{*} / U_{g}^{*}$ depends on the product $U_{g}^{*} \delta$. Fig. 2 shows $U_{n \max }^{*} / U_{g}^{*}$ variation with $U_{g}^{*} \delta$ which has been obtained numerically solving Eq. (34). It has been found that a good fitting law is

$\frac{U_{\eta \max }^{*}}{U_{g}^{*}}=c \approx \frac{2}{3}\left(2+e^{\left(-\delta U_{\mathrm{g}}^{*}\right)}\right)$ 


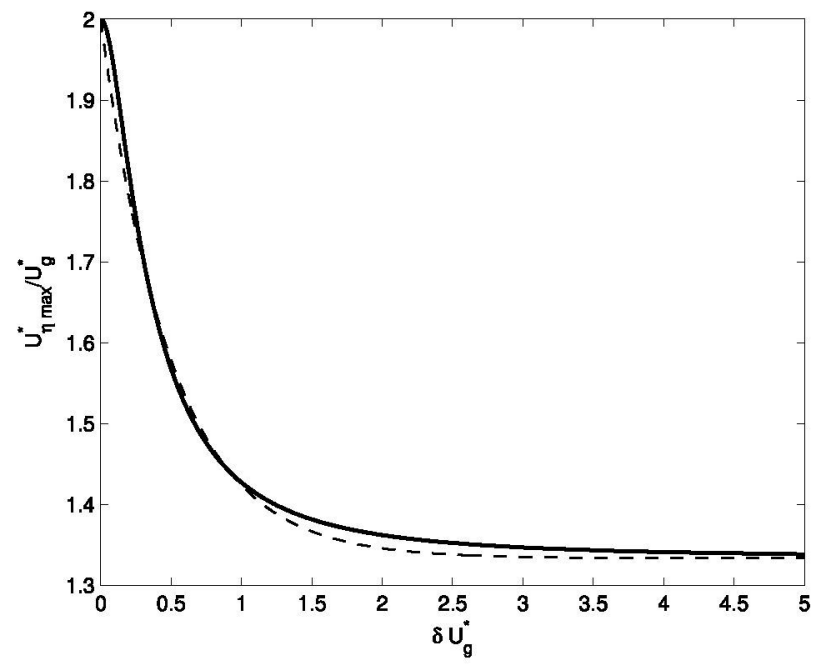

Fig. 2. $U_{n \max }^{*} / U_{\mathrm{g}}^{*}$ dependence with $\delta U_{\mathrm{g}}^{*}$. Solid line stands for numeric solution of Eq. (28) whereas dashed line shows the approximation given in Eq. (29).

where $c$ is a variable introduced for convenience. Substituting this law into Eq. (32), taking $U^{*}=U_{\eta \max }^{*}=c U_{g}^{*}$, it is found that

$$
\begin{aligned}
\eta_{\max } & =-\frac{a_{1}^{2}}{6 a_{3}} \frac{1}{1+\zeta / \zeta_{E}}\left(\left(\frac{4(c-1)}{c^{2}}\right) \frac{1}{1+\frac{16 c^{2} \delta^{2} m^{* 2}\left(\zeta+\zeta_{E}\right)^{2}}{a_{1}^{2}}}\right) \\
& =\eta_{\text {aero }} \eta_{\text {piezo }} \eta_{\text {cant }},
\end{aligned}
$$

where the value of $U_{g}^{*}=4 m^{*}\left(\zeta+\zeta_{E}\right) / a_{1}$ has been taken into account. This indicates that the maximum achievable efficiency depends on the geometry of the cross-section of the prism by means of an aerodynamic efficiency factor, namely $\eta_{\text {aero }}=-a_{1}^{2} /\left(6 a_{3}\right)$, on the ratio of mechanical damping to piezoelectric damping in a second efficiency factor $\eta_{\text {piezo }}=\left(1+\zeta / \zeta_{E}\right)^{-1}$ and, finally, on the cantilever effect which is defined by another efficiency factor which recovers the cantilever influence,

$\eta_{\text {cant }}=\left(\frac{4(c-1)}{c^{2}}\right) \frac{1}{1+\frac{16 c^{2} \delta^{2} m^{* 2}\left(\zeta+\xi_{\mathrm{E}}\right)^{2}}{a_{1}^{2}}}$.

Observe that $\eta_{\text {cant }} \leq 1$ for $\delta>0$ since $3 / 4 \leq 4(c-1) / c^{2} \leq 1$ in the range of interest $(4 / 3 \leq c \leq 2)$. In other words, there is a drop in $\eta_{\max }$ in the cantilever arrangement due to the induced rotation angle. Let us discuss qualitatively the role of the different parameters in $\eta_{\max }$ : it follows from Eq. (36) that it is beneficial to have high values of $a_{1}$, low values of $-a_{3}$, low $\zeta$, and low $\delta$. In addition, it can be seen that there must exist an optimal value of $\zeta_{E}$ since $\partial \eta_{\text {piezo }} / \partial \zeta_{E}>0$ and $\partial \eta_{\text {cant }} / \partial \zeta_{E}<0$.

Finally, it should be noted from Eq. (36) that maximum efficiency achievable is $-a_{1}^{2} /\left(6 a_{3}\right)$ since $\eta_{\text {piezo }}$ and $\eta_{\text {cant }}$ may achieve a theoretical value equal to one. Maximum power achievable would be limited by the size of the prism, fluid density, and flow speed, as can be deduced from Eq. (25), taking into account that $\eta=$ $-a_{1}^{2} /\left(6 a_{3}\right), P_{E \max }=\rho U^{3} D L a_{1}^{2} /\left(12 a_{3}\right)$.

\section{Model validation}

A detailed experimental campaign of piezoelectric energy harvesting from aero-elastic galloping was carried out by Zhao et al. [8]. The experimental arrangement consisted in a rigid square-section cylinder fixed to the free end of a cantilevered beam of length. For electricity conversion piezoelectric sheets were attached to the base of the beam. Table 1 lists the mechanical and piezoelectric parameters in their experiments.
Table 1

Physical properties in Zhao et al. [8] experiments.

Source: Values of aerodynamic coefficients $a_{1}$ and $a_{3}$ are taken from [16].

\begin{tabular}{lll}
\hline Parameter & Configuration 1 & Configuration 2 \\
\hline $\mathrm{m}(\mathrm{kg})$ & 0.029 & 0.029 \\
$\mathrm{k}(\mathrm{N} / \mathrm{m})$ & 381.6 & 381.6 \\
$C_{p}(\mathrm{nF})$ & 180 & 180 \\
$\theta(\mathrm{N} / \mathrm{V})$ & $3.73 \cdot 10^{-4}$ & $3.73 \cdot 10^{-4}$ \\
$R_{L}(\mathrm{k} \Omega)$ & 105 & 105 \\
$L_{b}(\mathrm{~m})$ & 0.15 & 0.15 \\
$D(\mathrm{~m})$ & 0.04 & 0.04 \\
$L(\mathrm{~m})$ & 0.15 & 0.10 \\
$\rho\left(\mathrm{kg} / \mathrm{m}^{3}\right)$ & 1.2 & 1.2 \\
$U(\mathrm{~m} / \mathrm{s})$ & $2-8$ & $2-8$ \\
$m^{*}$ & 107.6 & 161.5 \\
$\zeta$ & 0.005 & 0.004 \\
$\omega_{N}(\mathrm{rd} / \mathrm{s})$ & 53.7 & 53.7 \\
$\delta$ & 0.4 & 0.4 \\
$a_{1}$ & 2.3 & 2.3 \\
$a_{3}$ & -18 & -18 \\
\hline
\end{tabular}

In Fig. 3(a), a comparison between experimental results and analytical results (Eq. (31)) for the mean electrical power dissipated at the electrical resistance as a function of the wind speed is presented. Good agreement can be noted. It is also shown, for illustrative purposes, the analytical prediction obtained without considering the cantilever effect (that is, taking $\delta=0$ ). It is noteworthy that the mean electrical power is considerably less for the cantilevered arrangement (around 52\%, 46\% and 42\% less for $U=4,5$, and $6 \mathrm{~m} / \mathrm{s}$ respectively).

Fig. 3(b) shows the efficiency given by the theoretical model (Eq. (32)) along with experimental results. Again, the analytical prediction obtained without considering the cantilever effect is shown for completeness. As it can be seen, there is a drop in the efficiency for the cantilever arrangement with respect to the case of pure TG. In addition, the velocity at which the efficiency is maximum $U_{\eta \max }^{*}$ is lower than that of pure TG. It can also be noted that predictions given by Eqs. (35) $\left(U_{\eta \max }^{*} \approx 2.4\right)$ and $(36)\left(\eta_{\max } \approx\right.$ 0.009 ) are in reasonable agreement with experimental results. It is interesting to note that in this case $\eta_{\text {aero }}=0.049, \eta_{\text {piezo }}=0.36$ and $\eta_{\text {cant }}=0.48$ which shows that efficiency losses by cantilever arrangement are significant. Efficiency losses due to cantilever effect are significant because the presence of the cantilever adds an additional angle $\theta$ to the angle of attack, which results in lower achievable galloping amplitudes and less energy transfer. In addition, as already anticipated the cross-section geometry of the prism dictates the maximum achievable aeroelastic efficiency. Galloping response of a square prism is weak in comparison with other cross-section geometries like triangular, or D-type $[1,14]$.

Finally, Fig. 4(a) and (b) show experimental results from Zhao et al. [8] and analytical predictions for a configuration with a larger value of the mass ratio. Predictions for $U_{\eta \max }^{*}$ is 2.85 (Eq. (35)) and for $\eta_{\max } 0.005$ (Eq. (36)), which are in reasonable agreement with experimental results. It is worth noting that even at a relatively large value of $\delta$, where the hypothesis of low rotating angle and low angle of attack considered in the theoretical model may be more doubtful, the degree of agreement is fairly good. As predicted by Eq. (28), the wind speed at which galloping starts has been increased with respect to the case of lower $m^{*}$, and the harvested power has decreased (as can be deduced from Eq. (31)).

To see more clearly the precise effect of $\delta$, let us consider values of configuration and make $\delta$ a free parameter. Fig. 5 shows the amplitude of oscillations and electrical power as a function of $\delta$ and fluid velocity. As can be seen, for values of flow velocity close to that of starting galloping, $\delta$ barely affects the response; however, when flow velocity gets higher, amplitude growth with the reduced velocity $U^{*}$ is diminished with $\delta$, even getting into an asymptotic value as was anticipated in Eq. (29). The behavior of 
a

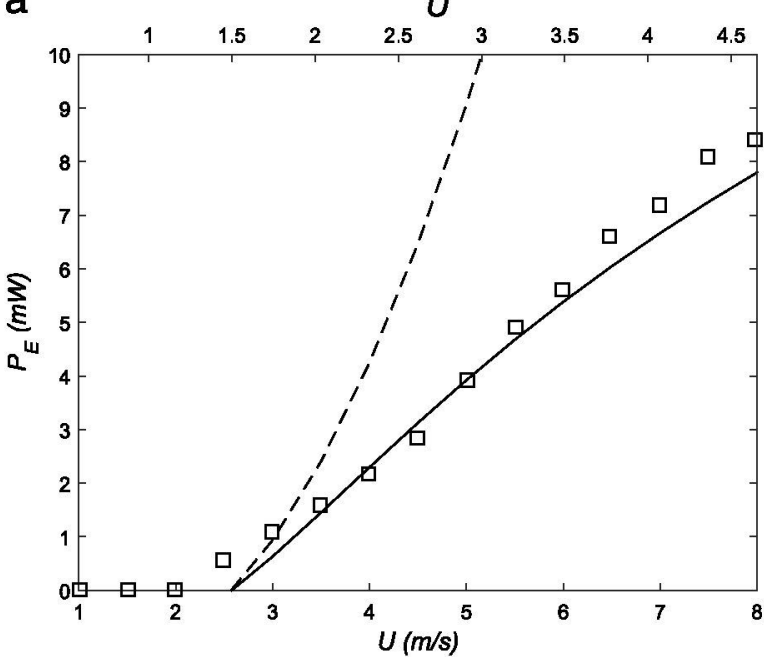

b

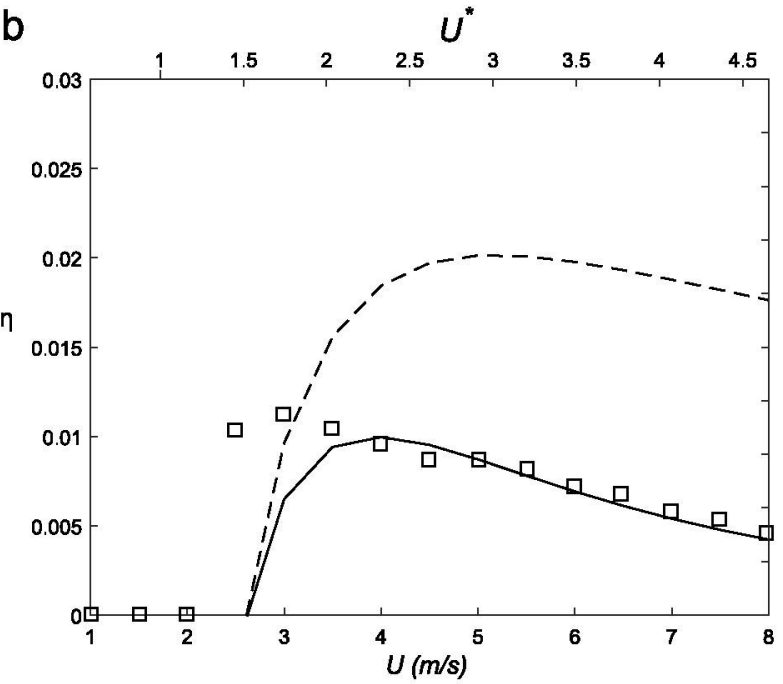

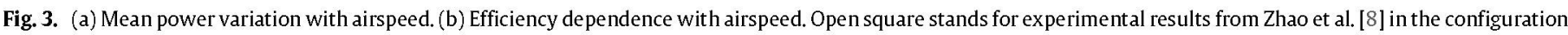

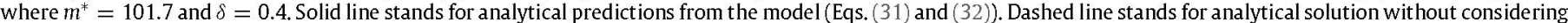
the cantilever aerodynamic effect (pure TG).
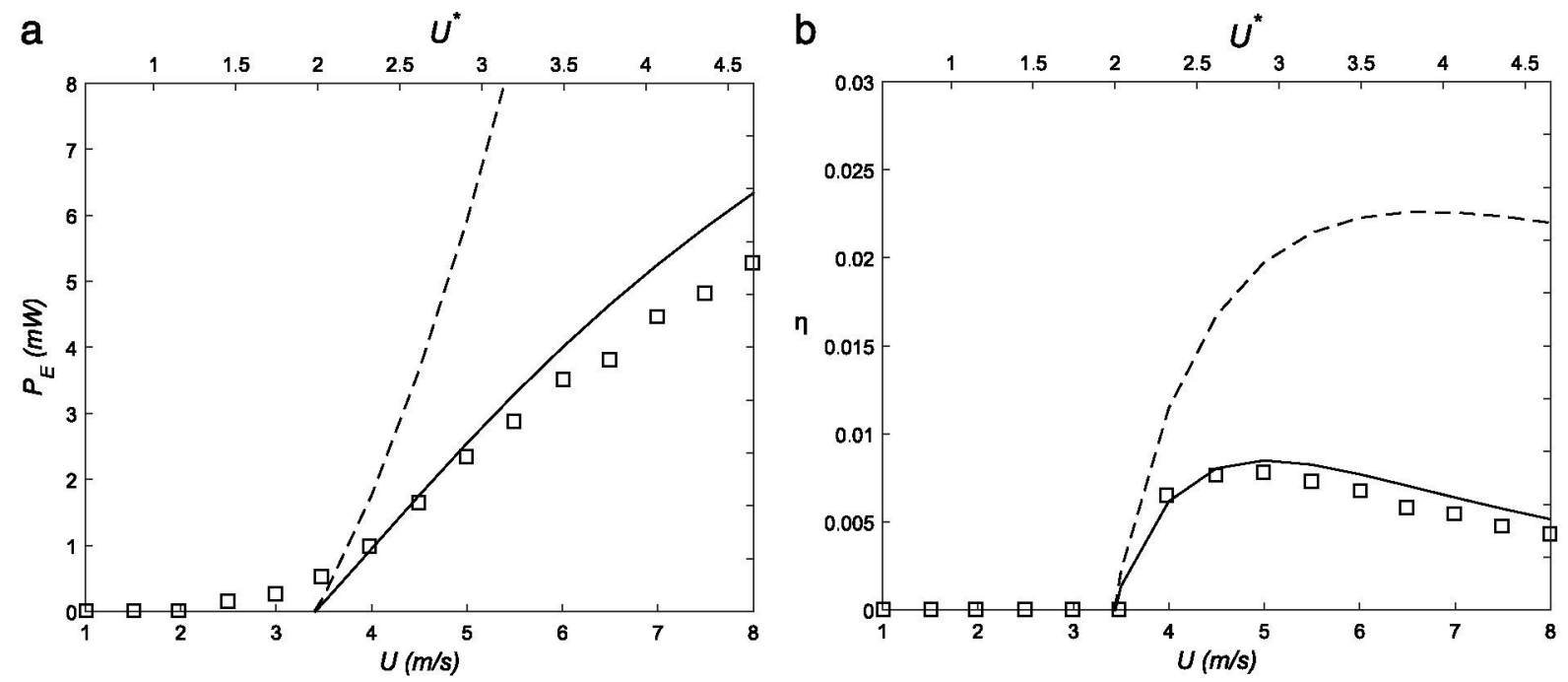

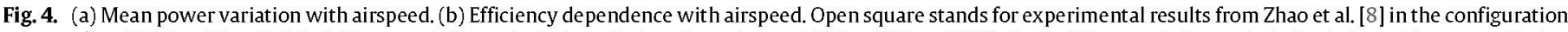

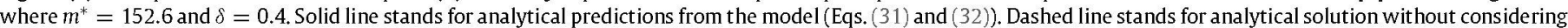
the cantilever aerodynamic effect (pure TG).

electrical power is qualitatively the same and it can be seen that, for a fixed wind speed, pure TG seems to be always a more convenient option than the cantilever one. This is because the cantilever always introduces an efficiency factor (see Eq. (37)) to the energy conversion system. Nevertheless, taking into account engineering design aspects, it seems clear that cantilever configurations can be easily implemented, with low mechanical parasitic damping and low costs, whereas pure TG requires a more sophisticated mounting system to maintain the attitude of the prism.

\section{Concluding remarks}

A theoretical model representing the dynamics of oscillatory motion of a piezo-galloping cantilever system coupled to a purely resistive energy harvesting circuit has been considered. A nonlinear quasi-steady approximation of the aerodynamic force has been utilized and the influence of the rotation angle due to the cantilever arrangement considered. An analytical solution has been given which has been compared favorably with experimental results from Zhao et al. [8]. This constitutes a theoretical guide to optimize the power by selecting appropriately the mechanical properties and tuning the electrical resistance. In addition, the model has been analyzed in some detail with the following findings:

- The electrical power output depends significantly on the length aspect of the cantilever $(\delta)$. The lower the length aspect of the beam the lower the level of energy harvesting. For very high values of mass ratio (typical for aeroelastic situations) electrical power output is proportional to $\left(1+\delta^{2} U^{* 2}\right)^{-1}$ (see Eq. (33)).

- The reduced velocity at which galloping oscillations starts (and electrical power output) depends slightly on $\delta$ and diminishes with respect to the value predicted by pure TG modeling.

- The electrical power output tends to an asymptotic finite value for a large reduced velocity $U^{*}$, whereas in pure TG the electrical power output grows without limit with $U^{*}$. This asymptotic value is due to the cantilever effect on galloping oscillation amplitude.

- The reduced velocity at which the efficiency is $\operatorname{maximum}\left(U_{\max }^{*}\right)$ depends on $\delta$. For very large beams $(\delta \longrightarrow 0)$ it is found that 

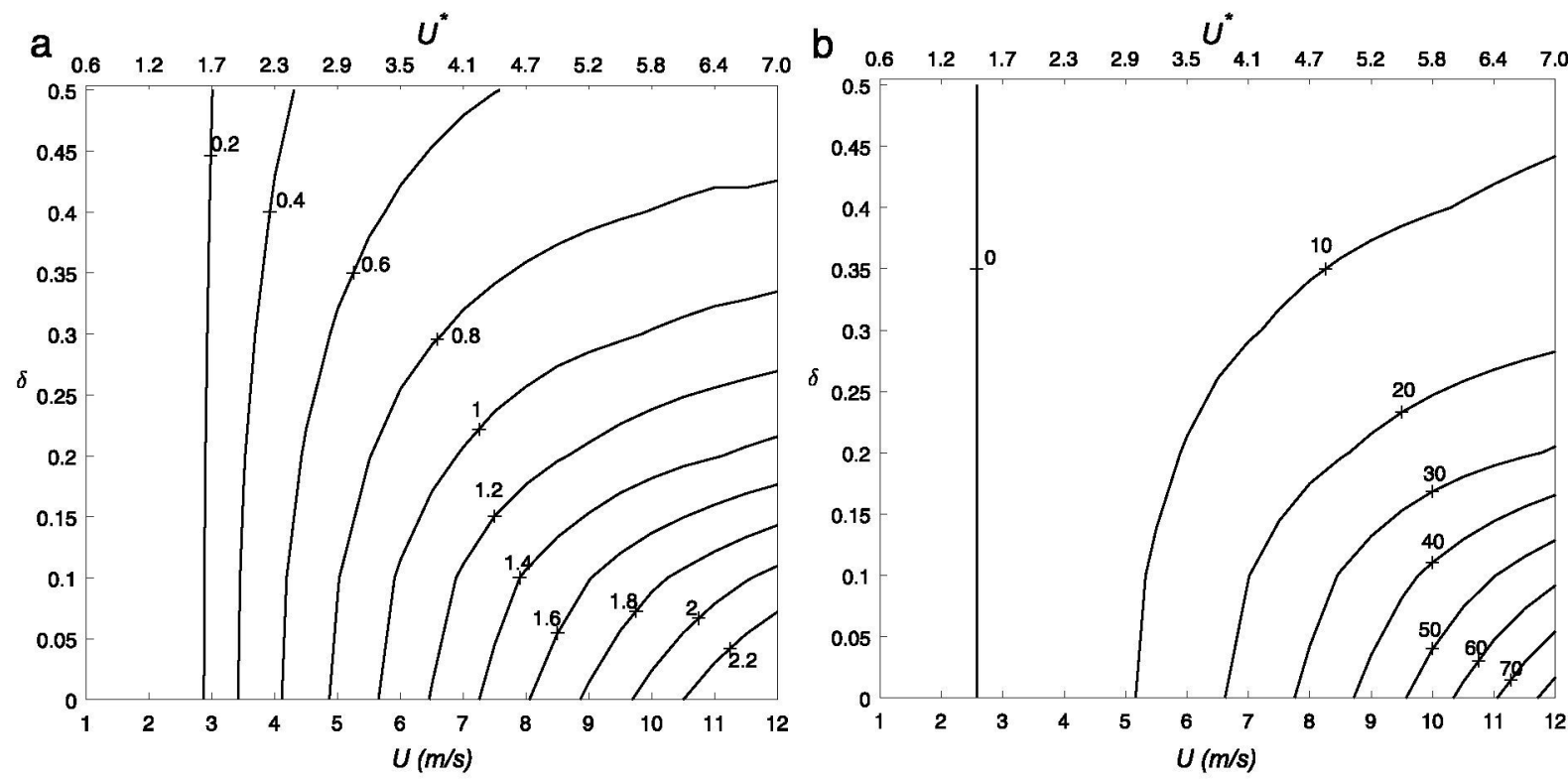

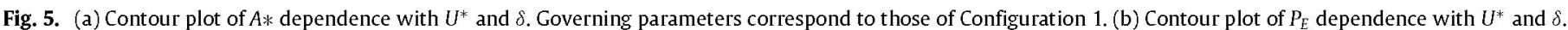
Governing parameters correspond to those of Configuration 1.

$U_{\max }^{*} \longrightarrow 2 U_{g}^{*}$, whereas for short beams $U_{\max }^{*}$ approaches to $4 U_{g}^{*} / 3$. Observe that in a real site with a certain environmental wind speed we would be able to adjust $U_{g}^{*}$ (by changing $m^{*}$ for example, see Eq. (28)) in order to work in the point of maximum efficiency.

- Maximum attainable efficiency diminishes with the length aspect ratio of the cantilever (see Eq. (37)). Efficiency losses by cantilever arrangement may be significant.

- With regards to the dynamics of the prism, the normalized amplitude of oscillations $A^{*}$ for cantilever galloping is lower than that of pure Transverse Galloping. Conversely, the normalized frequency of oscillations is larger. $A^{*}$ tends to an asymptotic finite value for a large reduced velocity $U^{*}$.

Some open questions derived from the present study require further insight, like the effect due to torsional bending of the cantilever, piezoelectric sheet size and placement, or the optimal cross-section geometry sections. In addition, in the present analysis the electrical load at which power is dissipated has been considered constant. It could be of interest to see if there exists an optimal electrical load for each flow speed in order to improve the power extracted. We are currently working on these questions.

\section{References}

[1] A. Barrero-Gil, A. Sanz-Andres, G. Alonso, Energy harvesting from transverse galloping, J. Sound Vib. 329 (14) (2010) 2873-2883.
[2] G. Parkinson, Phenomena and modelling of flow-induced vibrations of bluff bodies, Prog. Aerosp. Sci. 26 (2) (1989) 169-224.

[3] M.P. Paidoussis, J.P. Stuart, E. DE Langre, Fluid-Structure Interactions, Cambridge University Press, 2012.

[4] D. Vicente-Ludlam, A. Barrero-Gil, A. Velazquez, Optimal electromagnetic energy extraction from transverse galloping, J. Fluids Struct. 51 (2014) 281291.

[5] D. Vicente-Ludlam, A. Barrero-Gil, A. Velazquez, Enhanced mechanical energy extraction from transverse galloping using a dual mass system, J. Sound Vib. 339 (2015) 290303.

[6] J. Sirohi, R. Mahadik, Piezoelectric wind energy harvester for low-power sensors, J. Intell. Syst. Struct. 22 (2011) 2215

[7] J. Sirohi, R. Mahadik, Harvesting wind energy using a galloping piezoelectric beam, J. Vib. Acoust. 134 (1) (2012) 011009

[8] L. Zhao, L. Tang, Y. Yang, Comparison of modeling methods and parametric study for a piezoelectric wind energy harvester, Smart Mater. Struct. 22 (2013) 125003.

[9] Y. Yang, L. Zhao, L. Tang, Comparative study of tip cross-sections for efficient galloping energy harvesting, Appl. Phys. Lett. 102 (2013) 064105.

[10] J. Xu-Xu, A. Barrero-Gil, A. Velazquez, A theoretical study of the coupling between a vortex-induced vibration cylindrical resonator and an electromagnetic energy harvester, Smart Mater. Struct. 24 (2015) 115009.

[11] J.M. Kluger, F.C. Moon, R.H. Rand, Shape optimization of a blunt body Vibrowind galloping oscillator, J. Fluids Struct. 40 (2013) 185-200.

[12] L.N. Virgin, Introduction to Experimental Nonlinear Dynamics. A Case Study in Mechanical Vibration, Cambridge, 2000.

[13] R. Blevins, Flow-Induced Vibrations Van Nostrand Reinhold, Amsterdam, 1990.

[14] A.R. Bokaian, F. Geoola, On the cross flow response of cylindrical structures, in: Proc. Instn. Civ. Engrs, Part 2, 1983, Vol. 75, Sept., pp. 397-418.

[15] A. Ertuk, W. Vieira, C. De Marqui, D. Inman, On the energy harvesting potential of piezoaeroelastic systems, Appl. Phys. Lett. 96 (2010) 184103.

[16] M. Novak, H. Tanaka, Effect of turbulence on galloping instabilities, ASCE J. Eng. Mech. Div. 100 (1974) 27-47 